\title{
Uma análise do progresso científico da Cognição Social à luz da perspetiva do "novo Experimentalismo"
}

\author{
Leonel Garcia-Marques ${ }^{1}$ \& Teresa Garcia-Marques ${ }^{2}$ \\ ${ }^{1}$ Faculdade de Psicologia, Universidade de Lisboa \\ 2 ISPA- Instituto Universitário
}

\begin{abstract}
Resumo: A Cognição Social é uma abordagem conceptual e empírica que tenta explicar os fenómenos psicossociais através das estruturas e dos processos cognitivos que lhe são subjacentes. Esta abordagem com a sua génese nos anos oitenta, tem contribuído para a compreensão da mente do ser humano, tomando-o como um ser eminentemente social. Neste artigo procuramos debater a sua contribuição para o progresso científico na conceção da abordagem epistemológica do "novo experimentalismo", que caracteriza este progresso como o resultado da acumulação de "bons paradigmas experimentais". Exemplificamos o tipo de paradigmas experimentais, que se definem como "bons paradigmas" na Cognição Social, focando o paradigma das Correlações Ilusórias na perceção de grupos humanos; o paradigma da Mera-Exposição, o paradigma de Supressão de Pensamentos; e o paradigma do Estado de Espírito como Moderador do Processamento Cognitivo.
\end{abstract}

Palavras-chave: Novo experimentalismo; Cognição Social.

An analysis of the Scientific progress of Social Cognition in the light of the perspective of "new Experimentalism": Social Cognition is a conceptual and empirical approach that attempts to explain psychosocial phenomena through the structures and cognitive processes underlying it. This approach, with its genesis in the 1980s, has contributed to the understanding of the human mind taking it as eminently social. In this article, we discuss its contribution to scientific progress in the conception of the "new experimentalism" epistemological approach. New experimentalism characterizes scientific progress as the result of the accumulation of "good experimental paradigms". We offer a number of examples of what should be considered as "good paradigms" in social cognition, focusing on the paradigms of Illusory Correlations in the perception of human groups; the Mere-Exposure paradigm, the Suppressing Thoughts paradigm; and the Mood as Moderator of cognitive processing paradigm.

Keywords: New Experimentalism, Social Cognition

No tempo em que os animais (e as coisas) falavam connosco e nós eramos suficientemente ingénuos para o acreditar, o progresso científico estava garantido.

Tempos houve em que "progresso" fazia parte da própria noção de ciência. Nos nossos dias, contudo, em que a epistemologia da ciência se tornou central e inevitável, a ideia de progresso científico é mais crítica e menos consensual. Daí que num artigo que pretende debater os avanços da Cognição Social nos últimos anos, nos parece adequado incluir uma discussão sobre o que se entende por progresso científico. Por isso, depois de uma breve definição da disciplina da Cognição Social, discutiremos brevemente a noção de progresso e de avanços científicos, adotaremos as propostas do novo experimentalismo, e apresentaremos exemplos de progressos na Cognição Social segundo esta perspetiva.

\section{0 que é a Cognição Social}

A datação do início de uma abordagem e disciplina científica é sempre discutível. Mas uma data relativamente consensual para o início da disciplina Cognição-Social pode bem ser a publicação do livro "Person Memory" editado por Reid Hastie, Thomas M. Ostrom, Ebbe B. Ebbesen, Robert S. Wyer Jr., David L. Hamilton e Donal E. Carlston, em 1980.

Assim, a Cognição Social pode definir-se como uma abordagem conceptual e empírica que tenta explicar os fenómenos psicossociais através das estruturas e dos processos cognitivos que lhe são

\footnotetext{
${ }^{1}$ Morada para correspondência: Leonel Garcia-Marques, Faculdade de Psicologia da Universidade de Lisboa, Alameda da Universidade,
} 1649-013 Lisboa, Portugal. E-mail: garcia_marques@sapo.pt 
subjacentes (Hamilton et al., 1994). Hamilton et al. (1994), aliás, identificaram as quatro características fundamentais da Cognição Social. A saber:

a) A Cognição Social incide na investigação directa dos fundamentos cognitivos dos fenómenos sociais que aborda.

b) A Cognição Social adopta o modelo do processamento da informação como um meio de compreender os fenómenos sociais.

c) A Cognição Social pressupõe a identidade básica dos processos cognitivos subjacentes aos vários domínios substantivos da Psicologia.

d) A Cognição Social representa uma abordagem conceptual genérica e não apenas uma disciplina da ciência psicológica.

\section{O que deverá contar como "progresso científico"}

Bird (2007) sintetiza de forma bastante útil as várias concepções que existem de progresso científico. Agrupa-as em três grandes perspetivas: a perspetiva epistemológica, a perspetiva semântica e a perspetiva funcional. De uma perspetiva epistemológica, o progresso científico é visto como ocorrendo por acumulação de conhecimento (por exemplo, Sarton, 1936). Os que assumem uma perspetiva semântica percebem o progresso científico como ocorrendo por aproximação à verdade (por exemplo, Popper, 1963). Segundo a perspetiva funcionalista, o progresso científico dá-se pela resolução de desafios ou problemas científicos ou tecnológicos lançados dentro de uma dada comunidade científica. Sendo essa resolução de desafios ou de problemas científicos, realizada de acordo com os seus critérios de validação (por exemplo, Kuhn, 1970).

A refutação empírica da teoria mais "corroborada" da história da Ciência - a Mecânica Newtoniana - causou sérios problemas à primeira destas perspetivas e os argumentos sobre a incomensurabilidade dos paradigmas que se sucedem no desenvolvimento ciência levantou objeções difíceis de ultrapassar à segunda perspetiva. Por seu lado, a perspetiva funcionalista ao caracterizar a investigação científica como um empreendimento eminentemente "técnico" levado a cabo por especialistas (investigadores) e cujos progressos são indecifráveis para os não iniciados, tem um difícil mapeamento no mundo real, parecendo definir o sucesso da ciência como algo que ocorre com um estatuto semelhante ao de um milagre (Putnam, 1975).

O novo experimentalismo. Mais recentemente, o "novo experimentalismo" (Mayo, 1996) veio oferecer uma nova e mais satisfatória caracterização do progresso científico que agrega o que cada uma das perspetivas anteriores tem de melhor. Assim, a perspetiva do "novo experimentalismo" diz-nos que o progresso da Ciência se deve não à acumulação de conhecimento ou à aproximação da verdade, nem apenas por resolução de desafios, mas à criação de restrições à construção teórica. Assim, o progresso científico de uma disciplina deve ser avaliado pelo número e qualidade de restrições empíricas aceites na construção teórica. Estas restrições são criadas pelos resultados de experimentos e paradigmas experimentais essenciais. Estes "novos dados" têm, a partir do momento em que são conhecidos, de ser considerados por qualquer nova teoria que pretenda ser levada a sério. Assim, a perspetiva do "novo experimentalismo" centra-se na ideia de "bons" experimentos e paradigmas experimentais, caracterizando o progresso da ciência como o resultado da acumulação desses experimentos e paradigmas numa dada disciplina. Esta perspetiva define os "bons" paradigmas experimentais atribuindo-lhes as seguintes características:

a) desenvolvem-se em contextos adequados de correção de erros. Ou seja, os erros produzidos por um bom paradigma experimental têm a capacidade de promover o progresso científico e a aprendizagem. Isto porque a própria natureza do contexto em que emergiram os erros permite a sua identificação e correção.

b) produzem resultados que são apenas fracamente dependentes de uma qualquer teoria. Ou seja, dado um mínimo de pressupostos ontológicos compartilhados na respectiva comunidade científica, os resultados dos bons paradigmas experimentais, após a necessária verificação, correção e replicação, tendem a ser aceites como válidos, mesmo a partir de pontos de vista teóricos antagónicos.

c) testam hipóteses que poderiam facilmente ser falsas, ou seja, hipóteses muito específicas que proíbem muitos estados alternativos e plausíveis da natureza.

d) representam testes severos para as hipóteses específicas que abordam. Isto é importante porque mesmo uma hipótese específica pode ser testada em outro tipo de contexto mais permissivo. Em tais contextos, resultados positivos para as hipóteses testadas podem ser obtidos com grande probabilidade mesmo quando essas hipóteses são falsas (ver Roberts \& Pashler, 2000).

\section{O “progresso científico” da Cognição Social na perspetiva do novo experimentalismo}

Para ilustrar o progresso da Cognição Social enquanto abordagem científica, propomo-nos, neste artigo, a 
analisar alguns dos seus paradigmas. Ilustraremos a natureza de paradigmas encontrados quer na fundação da abordagem quer no seu desenvolvimento. Assim seleccionámos dois paradigmas que foram originados noutras abordagens e que, de certo modo, já "representavam" a abordagem da Cognição Social antes de esta existir: o paradigma de correlações ilusórias na perceção de grupos humanos (Hamilton \& Gifford, 1976), e o paradigma de mera exposição (Zajonc, 1968); e dois paradigmas mais recentes que mantiveram vibrante a Cognição Social e a estenderam a novos domínios: o paradigma da supressão de pensamentos (Wegner et al., 1987), e o paradigma dos efeitos cognitivos do estado de espírito (Worth \& Mackie, 1987).

O paradigma de Correlações Ilusórias na Perceção de Grupos Humanos. A correlação ilusória é um enviesamento que consiste em perceber uma correlação entre duas variáveis que na realidade não estão correlacionadas entre si ou que estão menos correlacionadas do que o relatado nos julgamentos (Chapman, 1967). Na perceção de categorias sociais, o efeito de correlações ilusórias corresponde classicamente a julgar menos favoravelmente grupos minoritários do que maioritários quando a maior parte dos comportamentos que os membros de ambos praticam são positivos e com igual prevalência. Mais concretamente, Hamilton e Gifford (1976) apresentaram aos seus participantes 39 comportamentos de membros de dois grupos. Cada comportamento era atribuído a um indivíduo diferente. Desses 39 comportamentos, 26 foram praticados por membros do grupo A, e 13 do grupo B; 27 comportamentos eram positivos e 12 negativos. Não havia, contudo, relação entre a pertença grupal e probabilidade de praticar um comportamento positivo, já que aos membros do grupo A foram atribuídos 18 comportamentos positivos e 8 negativos e ao grupo B foram atribuídos exactamente metade de cada (9 positivos e 4 negativos). No entanto, os participantes avaliaram o grupo A mais positivamente do que o grupo B numa série de medidas dependentes.

Este efeito despertou grande interesse porque as abordagens anteriores ao estereótipo e preconceito ofereciam explicações baseadas em motivações particulares ou decorrentes de uma personalidade neurótica, de pressões culturais e/ou do exagero de um fundo de verdade (ver Ashmore \& DelBoca, 1981), e caracterizavam-nos como uma forma inferior de raciocínio. Contudo, o efeito de correlações ilusórias foi obtido, neste caso, com participantes sem patologias particulares, com grupos-alvo artificiais e arbitrários (portanto sem pressões culturais ou motivações particulares) e sem qualquer fundo de verdade (os grupos maioritário e minoritário praticavam a mesma proporção de comportamentos positivos e negativos). E ainda assim, o minoritário grupo B era discriminado relativamente ao maioritário grupo A. Daqui decorre que as causas mais importantes apontadas anteriormente como explicação para a emergência de estereótipos negativos, preconceitos e discriminação não são, na verdade, necessárias para a sua ocorrência. E este efeito de correlações ilusórias não parece corresponder a nenhuma forma sui generis ou inferior de raciocínio, pelo menos no sentido em que ocorre igualmente com julgamentos e estimativas em domínios não sociais (Chapman, 1967).

Hamilton e Gifford (1976) explicaram o efeito de correlações ilusórias em função da dupla assimetria presente nas dimensões subjacentes ao conjunto dos comportamentos (pertença grupal e valência). Esta dupla assimetria faz com que a associação da pertença grupal do grupo minoritário com os comportamentos de valência mais rara (na maior parte dos casos, os comportamentos negativos) seja duplamente infrequente e, portanto, particularmente distintiva e saliente. Por isso, tal associação tenderá a ser sobre-aprendida. Quer dizer, melhor codificada, mais facilmente recuperada ou recordada, e a afectar os julgamentos desproporcionadamente.

O paradigma de correlações ilusórias na perceção de grupos humanos possui, na nossa perspetiva, os atributos essenciais de um "bom paradigma experimental", vejamos porquê.

\section{Hamilton e Gifford (1976) como um "Bom Paradigma Experimental".}

a) $O$ paradigma de Hamilton e Gifford (1976) desenvolveu-se em contextos adequados de correção de erros. Por exemplo, Hamilton, Dugan e Trolier (1985) encontraram uma correlação entre o número de comportamentos duplamente infrequentes recordados e a magnitude das correlações ilusórias. Tal correlação corroborava claramente a explicação original de Hamilton e Gifford (1976) mas como apenas foi replicada pela investigação subsequente em circunstâncias muito específicas (McConnell, Sherman \& Hamilton, 1994a), esse efeito deixou de ser utilizado para caracterizar os efeitos da dupla infrequência no julgamento de grupos humanos (Stroessner \& Plaks, 2001).

b) $O$ paradigma de Hamilton e Gifford (1976) produz resultados que são apenas fracamente dependentes de uma qualquer teoria. Por exemplo, enquanto Hamilton e Gifford (1976) situam a sua explicação destes efeitos na sobre-aprendizagem dos itens duplamente infrequentes, Fiedler (1991) situaa precisamente na aprendizagem deficiente desses itens mais vulneráveis aos efeitos de regressão e à entropia. No entanto, ambas as perspetivas convergem na realidade dos resultados obtidos por Hamilton e 
Gifford (1976). Mais, o número de explicações diferentes destes mesmos efeitos não tem parado de aumentar (para um exemplo recente que parte de uma teoria geral de categorização, ver Sherman et al., 2009).

c) O paradigma de Hamilton e Gifford (1976) testa hipóteses muito específicas. Apesar do efeito de correlações ilusórias em si ser pouco específico (ver Chapman, 1969), Hamilton e Gifford (1976) previram e obtiveram um tipo de correlação ilusória específico (desvalorização avaliativa do grupo minoritário) e a investigação posterior tendeu a tornar ainda mais específicas as condições em que o efeito é mais e menos passível de ser obtido (McConnell et al., 1994a, 1994b; Sanbonmatsu et al., 1987).

d) O paradigma de Hamilton e Gifford (1976) representa um teste severo de uma hipótese específica. No primeiro dos estudos originais, a desvalorização do grupo minoritário poderia ter beneficiado de um dos mais robustos efeitos encontrados em Psicologia - o efeito de mera exposição (ver adiante). Quer dizer, um grupo-alvo apresentado mais frequentemente tende a ser avaliado mais positivamente do que um alvo apresentado menos frequentemente (Zajonc, 1980). Por isso, neste caso, a dupla distintividade e o efeito de mera exposição convergem na sobre-valorização relativa do grupo maioritário e na desvalorização relativa do minoritário. No entanto, no segundo dos estudos originais, Hamilton e Gifford (1976) opuseram o efeito de dupla distintividade ao efeito de mera exposição, fazendo com que a maior parte dos comportamentos apresentados fosse negativo e, assim, os comportamentos duplamente distintivos passassem a ser positivos. Mas o efeito de correlações ilusórias manteve-se. Quer dizer, os itens duplamente distintivos (neste caso, os comportamentos positivos do grupo minoritário) tiveram um peso desproporcionado na avaliação desse grupo.

Em suma, podemos considerar que o paradigma experimental construído por Hamilton e Gifford (1976) é um bom paradigma experimental na acepção dada pelo novo experimentalismo. Essa qualidade, permitiu que o paradigma ganhasse vida própria contribuindo para a criação da própria Cognição Social e seja hoje tomado como uma restrição inevitável à teorização sobre a perceção de grupos humanos. Daí que qualquer nova abordagem sobre o tema que queira ser levada a sério tem de ser capaz de oferecer uma explicação da dupla distintividade na perceção de grupos humanos.

0 paradigma de Mera-exposição. 0 paradigma experimental da mera-exposição, desenvolvido por Robert Zajonc, antecede o nascimento da cognição social, mas vem a ser totalmente integrado nesta abordagem por satisfazer os seus objectivos. Trata-se de um dos paradigmas mais citados de todos os tempos no campo da psicologia em geral (ver Bornstein, 1989) e desenvolve-se em torno do esclarecimento do significado e limites das primeiras evidências do efeito (já obtidas por outros autores, ver Bornstein, 1989) que, no entanto, não deixaram claro o facto de se tratar de um efeito associado a uma "mera exposição" (i.e., apenas perceção do estímulo). Em 1968, Zajonc verifica que a mera familiaridade de um estímulo (por encontro prévio como mesmo) tem impacto nos julgamentos afetivos (preferência) dos mesmos e em 1980 Zajonc argumenta que tal acontece de forma independente da recordação explícita desses mesmos estímulos. Na primeira versão experimental do paradigma a garantir a mera exposição, Zajonc (1968) apresenta aos participantes um conjunto de caracteres chineses com vista a que se familiarizem com os mesmos. Nesta fase de familiarização alguns dos caracteres eram repetidos de 1 a 25 vezes, de forma a induzir diferentes níveis de familiaridade. Numa segunda fase, Zajonc pediu aos participantes para avaliarem o significado bom-mau (numa escala de 0-mau a 6-bom) de cada caracter, apresentando-lhes uma lista de caracteres repetidos misturados com novos exemplares. Os dados demonstram uma tendência para avaliar os caracteres como tão mais positivamente quanto mais foram repetidos. Em 1980 Zajonc analisa vários estudos (e.g., Matlin, 1971; Moreland \& Zajonc, 1977) que replicam o estudo dos caracteres chineses e acrescenta uma terceira fase, na qual se apresentou aos participantes a mesma lista com o objectivo de identificarem os caracteres com os quais tinham sido familiarizados. Os dados analisados pelo LISREL III) sugerem que a avaliação afectiva influenciava a memoria (.21) tanto quanto a experiência subjetiva de repetição influenciava o julgamento afetivo (.14), e que existia uma relação forte entre a repetição e a avaliação afectiva independente da sua recordação (na ordem de .66). A conclusão será a de que o julgamento afectivo e a recordação se baseiam em duas características distintas do processamento do estímulo: "preferanda" e "discriminanda" (Zajonc 1980). 0 que mais tarde fica a ser conhecido como paradigma de mera exposição é, porém, apenas o efeito da repetição nos julgamentos/manifestações de preferência, identificado em 1968, não sendo este necessariamente associado à dissociação encontrada entre uma medida cognitiva e afectiva. É assim que o efeito é: a) replicado com os materiais mais diversos que se possam imaginar (figuras geométricas, caracteres chineses, faces humanas, pessoas, produtos, etc.); b) replicado com diferentes tipos de participante, como estudantes, professores, crianças e até com animais (e.g., galinhas); e c) utilizado para explicar uma grande variedade de fenómenos. Zajonc (1968), é citado em estudos que se centram na preferência por uma grande variedade de alvos. É, deste modo, citado em estudos sobre alimentação para 
explicar a preferência por alimentos, em estudos de atração interpessoal para explicar a atracão por um dado alvo, em estudos de comportamentos aditivos, em estudos sobre música, publicidade, etc. (ver metaanálise de Bornstein, 1989).

Os resultados iniciais de Zajonc suscitaram um enorme interesse na comunidade científica não por causa do efeito de mera exposição em si, mas porque estes traziam para o campo do testável uma forma de abordar a relação entre os afetos e a cognição. E é por isso que o artigo de 1980 ganhou um prémio e tem até hoje em dia um dos maiores números de citações verificado. Neste aspecto, o que parece ser relevante para o paradigma não é apenas os efeitos sobre a medida de afeto utilizada, mas, igualmente, a sua independência deste efeito, no impacto que a manipulação tem numa medida de memória. Tal permitia identificar a mera exposição como um efeito puramente afetivo. É com base nesta dissociação que Zajonc argumenta em favor da hipótese de primazia afetiva (Zajonc, 1980). Na sua perspetiva, o efeito de mera exposição suporta a ideia de que as avaliações, tidas como uma resposta afetiva, são ativadas face a uma repetição do estímulo, mesmo quando esta repetição não tem impacto numa medida cognitiva como a recordação. Isto é, a resposta afectiva é activada de forma independente e em primeiro lugar do que a cognição. Dados subsequentes vêm dar suporte a esta hipótese ao sugerirem que não existe relação entre uma medida de memória e uma medida de preferência (e.g., Moreland \& Zajonc, 1977) e que o facto de se tornar os estímulos inconscientes (apresentação subliminar) teria um impacto negativo da recordação, mas mantinha intactos os efeitos de mera exposição (Murphy \& Zajonc, 1993).

A proliferação de estudos que focam o efeito de mera exposição, resultantes da procura dos seus limites e de testes à sua explicação, permitiu uma clara evolução deste paradigma, esclarecendo os seus contornos. Tal confere ao paradigma de mera-exposição, em nossa opinião, as características de um "bom paradigma experimental".

\section{Zajonc, 1968, como um "Bom Paradigma Experimental".}

a) 0 paradigma de mera exposição desenvolveu-se num contexto adequado de correção de erros. 0 efeito foi originalmente definido como puramente afectivo, sugerindo uma independência de efeitos de aprendizagem/memória. No entanto, este pressuposto vem a ser desafiado de diferentes formas em estudos subsequentes. Estes desafios envolvem a crítica às medidas de afeto e cognição utilizadas, levando à necessidade de se alterarem as suas operacionalizações. Se inicialmente o afeto se operacionalizou na categorização do significado do estímulo como Bom vs. Mau (entendido por alguns como refletindo apenas uma dimensão cognitiva), rapidamente evolui para escalas de preferência (gosto vs. não-gosto) e até medidas menos sujeitas a "interferência cognitiva" como a respostas dos músculos faciais (Harmon-Jones \& Allen, 2001). Estas sugerem uma maior ativação do músculo zigomático na presença de estímulos familiares. Por outro lado, autores como Lazarus (1984) chamam desde cedo atenção para o facto de cognição não poder ser reduzida a reconhecimento, tornando clara a existência de fenómenos cognitivos que não espelham necessariamente a memória. Se em 1968 e 1980 poderia fazer sentido pensar que a ausência de reconhecimento implica ausência de mecanismos cognitivos, atualmente sabe-se que o reconhecimento não é necessário e que a repetição tem influência em tarefas como identificação perceptiva, decisões lexicais, e complemento de fragmentos de palavras que se apresentam como testes indirectos de memória (e.g., Richardson-Klavehn \& Bjork 1988).

b) O paradigma de mera exposição produz resultados que são claramente independentes da teoria que o gerou. Os dados que foram gerados sobre o pressuposto de independência da cognição e afeto são atualmente integrados em qualquer teoria que não assuma os afetos como ancorados no processamento consciente das características "semânticas" de um estímulo. Assim, o fenómeno da mera exposição é integrado como evidência de que o ser humano responde a estímulos do seu ambiente que não acedem necessariamente à sua consciência, e que essas respostas têm por isso uma natureza mais próxima do tipo de sensações, sentimentos e afetos (ver, por exemplo, Chaiken \& Trope, 1999). 0 fenómeno é igualmente integrado nas abordagens que assumem que a perceção de um estímulo implica a ativação de mecanismos fisiológicos até ao momento apenas associado à dimensão afetiva. Estas abordagens corporalistas (embodiment; ver Barsalou, 2003) sugerem que o sentir é parte do compreender, não dissociando a resposta afectiva da cognitiva como sugerido por Zajonc (1980). Por outro lado, o fenómeno de mera exposição é integrado em explicações do mecanismo mnésico, sendo interpretado como evidência da memória implícita. Um exemplo será a abordagem apresentada por Bornstein e D'Agostino (1992) que faz uso do efeito de mera exposição e da falta de relação entre as medidas afetivas e de memória para, baseando-se nos trabalhos de Larry Jacoby (Jacoby, 1991), sugerirem que a fluência com que processamos um estímulo pode ser atribuída a julgamentos de memória e/ou preferência, em função da questão colocada ao participante.

c) O paradigma de ME testa hipóteses muito específicas. 0 paradigma pretendeu responder à simples questão de se a repetição (já conhecida nos seus efeitos de aprendizagem) é primeiramente, e de forma 
independente, "sentida". Assim, esperava-se um e apenas um padrão de resultados: que o afeto fosse mais positivo quanto mais familiar fosse o estímulo, mesmo se essa familiaridade não fosse detectada em medidas de memória. Investigação posterior tendeu a tornar mais específicas as condições em que o efeito é mais e menos passível de ser obtido, focando as condições associadas à apresentação prévia (tipo de repetição, tempo de apresentação, heterogeneidade da apresentação, etc.), tipologia de estímulos (neutros, afetivos, imagens, palavras, etc.), condições de apresentação, condições do individuo, etc. (para uma revisão, ver Bornstein, 1989).

d) O paradigma de ME representa um teste severo de uma hipótese específica. Zajonc, em 1968, estabelece condições severas ao teste do efeito da repetição no julgamento afetivo ao controlar muitos outros fatores que explicavam o efeito, por usar estímulos totalmente não familiares e sem significado prévio para os participantes experimentais. No primeiro dos estudos originais não estaria claro (dado o estado do conhecimento) que a repetição seria detetada por um mecanismo afetivo de forma "independente" à aprendizagem (ou à consciência de aprendizagem), sendo o teste com os estudos de Murphy e Zajonc, (1993) o mais severo de todos por tornar a familiarização inconsciente.

Em suma, o paradigma experimental da ME tem todas as características de um bom paradigma. Parecem ser estas as que permitem que este paradigma tenha não apenas uma, mas muitas vidas próprias, servindo de âncora a várias abordagens teóricas, de diferentes campos de estudo, e tornando o artigo de Zajonc de 1980 um dos mais citados de sempre da história da Psicologia. Assim, este paradigma não só contribuiu para a criação da própria Cognição Social como influencia várias outras abordagens e campos de estudo (com especial ênfase para o estudo de memória em Psicologia Cognitiva).

0 paradigma de supressão de pensamentos. 0 estudo da eficácia e das consequências da supressão de pensamentos enquanto forma de controlo mental foi trazida para o laboratório por Wegner et al. (1987). Wegner et al. (1987) pediram aos participantes que reportassem os seus pensamentos em voz alta durante um período de 5 minutos, pedindo-lhes que tentassem pensar num "urso branco" (fase de expressão) ou que evitassem pensamentos acerca de um "urso branco" (fase de supressão). O procedimento era executado uma segunda vez; os participantes que já tinham passado pela fase de expressão passavam agora para a fase de supressão e vice-versa. Dizendo de outra forma, foram constituídos dois grupos de participantes diferindo apenas na ordem em que passavam pelas duas fases, o grupo Supressão-Expressão, e o grupo Expressão-Supressão. Em ambas as fases (de expressão e supressão), contudo, foi pedido adicionalmente aos participantes que tocassem uma campainha cada vez que mencionassem ou pensassem sobre um urso branco. Os resultados deste estudo revelaram um efeito de ricochete; isto é, uma maior frequência de referências ao urso branco (a principal medida dependente) durante o período de expressão para o grupo Supressão-Expressão do que para o grupo Expressão-Supressão. Ou seja, após um período de supressão de pensamentos com um determinado conteúdo, esses pensamentos tornam-se mais insistentes do que se a supressão não tivesse (ainda) ocorrido.

Estes resultados suscitaram um enorme interesse na comunidade científica porque traziam para o campo do testável, preocupações antigas sobre os limites do controlo mental (por exemplo, Freud, 1900/1913) e ofereciam um complemento indispensável às teorias sobre os automatismos cognitivos. Na verdade, frequentemente, os processos automáticos só se tornam passíveis de estudo quando o controlo mental falha (ver, por exemplo, Jacoby, 1991).

0 interesse suscitado por estes resultados levou a que o paradigma inicial de Wegner et al. (1987) desse origem ao estudo mais aprofundado do controlo mental em laboratório. Tal estudo levou quer à descoberta de um grande número de outras consequências da supressão do pensamento para além do efeito de ricochete, como ao reconhecimento da importância do controlo para a compreensão de um grande número de domínios da vida mental que vão desde a influência dos estereótipos (Macrae et al., 1998) até às insónias, passando pelo golfe (para uma revisão, Wenzlaff \& Wegner, 2000).

Na explicação deste e de outros efeitos, Wegner desenvolveu o modelo irónico de controlo mental (Wegner, 1994). De acordo com este modelo, as tentativas de supressão de um dado conteúdo da consciência desencadeiam dois processos distintivos que funcionam em tandem, mas de cuja interação podem resultar efeitos irónicos. 0 primeiro destes processos é o processo automático de monitorização que detecta eventuais intrusões do pensamento indesejado. Em caso positivo, é desencadeado o segundo processo, que é um controlado de busca de pensamentos de substituição, o chamado processo operativo. De acordo com esta explicação, os efeitos de ricochete devem-se quer à natureza automática, quer ao funcionamento irónico do processo de monitorização. Como o processo de monitorização é de natureza automática, não é facilmente desativado de modo deliberado e pode, por isso mesmo, continuar a ocorrer muito após qualquer tentativa de supressão. E como o simples escrutinar da consciência em busca da presença dos pensamentos a suprimir pode trazer esses mesmos pensamentos à consciência, o processo 
de monitorização pode continuar a tentar "resolver" um problema já resolvido e por isso pode, ironicamente, voltar a criá-lo - daí resultando o efeito de ricochete.

O paradigma de supressão tem, em nossa opinião, as características de um "bom paradigma experimental"; vejamos porquê.

\section{Wegner et al. (1987) como um "Bom Paradigma Experimental".}

a) O paradigma de Wagner et al. (1987) desenvolveu-se em contextos adequados de correção de erros. Por exemplo, os estudos iniciais confundiam aprendizagem com os efeitos da supressão, por se comparar o controlo mental exercido nas fases de expressão entre os grupos Expressão-Supressão e SupressãoExpressão. Assim, o aumento do número de menções ao conteúdo a suprimir na fase de expressão do segundo grupo poder-se-ia dever quer ao efeito da supressão quer ao efeito da aprendizagem. De facto, como se pretendia que os participantes se concentrassem nos anteriores conteúdos a suprimir, mais menções poderiam significar apenas melhor domínio da tarefa (Clarke et al., 1991). Várias soluções foram desenhadas e utilizadas para corrigir rapidamente este problema. Por exemplo, Wegner e Gold (1995) introduziram uma fase inicial de Expressão comum aos dois grupos do delineamento, de modo a que os grupos passassem a ser Expressão-Expressão-Supressão e Expressão-Expressão-Expressão, em que a comparação fundamental é feita na terceira fase.

b) O paradigma de Wagner et al. (1987) produz resultados que são apenas fracamente dependentes de uma qualquer teoria. Por exemplo, Förster e Liberman (2004) apresentam o modelo MIMO, um modelo explicativo alternativo ao modelo de Wegner. Este modelo defende que a dificuldade sentida durante o envolvimento em processos de supressão (a supressão envolve esforço e nunca é completamente bemsucedida) leva à atribuição de motivação para exprimir esse constructo mental. Em consequência desta inferência, a expressão do conteúdo a suprimir torna-se desejável e o objectivo de o exprimir ganha importância motivacional ao ter sido contrariado durante a supressão. 0 efeito de ricochete seria assim a tentativa de satisfazer uma motivação anteriormente bloqueada. Percebe-se, assim, como esta explicação é completamente diferente do modelo irónico de Wegner (1994). No entanto, a realidade do efeito de ricochete em si não é, de forma alguma, posta em causa.

c) O paradigma de Wagner et al. (1987) testa hipóteses muito específicas. 0 paradigma de Wegner et al. (1987) estuda os efeitos de uma tarefa de supressão. De um certo ponto de vista, seria impossível que uma determinada tarefa não produzisse efeitos residuais em tarefas semelhantes que se seguissem. Seria, portanto, provável que se encontrassem diferenças no conteúdo das verbalizações dos dois grupos em contraste. Mas o paradigma de Wegner et al. (1987) explora um tipo muito específico de verbalizações - as menções do conteúdo anteriormente suprimido.

d) O paradigma de Wagner et al. (1987) representa um teste severo de uma hipótese específica. De facto, o paradigma de Wegner et al. (1987) deteta diferenças no número de menções de um conteúdo que os participantes são encorajados a expressar (antes ou depois de uma fase de supressão). A hipótese poderse-ia facilmente não verificar em virtude do grupo de controlo (Expressão-Supressão) ser, tal como o grupo Experimental (Supressão-Expressão), instruído a produzir exatamente as menções ao conteúdo suprimido ou a suprimir quando a hipótese é a de que esses conteúdos serão mais frequentemente produzidos no grupo experimental. Mais ainda, numa modificação do paradigma original de Wegner et al. (1987), em que se examina a acessibilidade dos conteúdos a suprimir durante a própria supressão, essa acessibilidade é aferida por comparação com uma condição em que os participantes são instados a concentrarem-se nesses mesmos conteúdos (Wegner \& Erber, 1992).

Em suma, podemos considerar que o paradigma experimental construído por Wagner et al. (1987) é um bom paradigma experimental na aceção dada pelo novo experimentalismo. Essa qualidade permitiu que o paradigma ganhasse vida própria, contribuindo para o desenvolvimento da Cognição Social, e sendo hoje tomado como uma restrição obrigatória à teorização sobre o controlo mental. Daí que qualquer nova abordagem sobre o tema que queira ser mais do que uma fantasia tem de provar o seu mérito, oferecendo uma explicação dos efeitos da supressão de pensamentos.

O paradigma de Mood como moderador do processamento cognitivo. Worth e Mackie, em 1987, baseando-se em estudos prévios sobre o efeito que o estado de espírito exerce sobre o funcionamento cognitivo, postulam e testam a hipótese de que os estados afectivos positivos induziriam os indivíduos a processar mais superficialmente a informação recebida. Contrariamente, os estados afetivos negativos, em vez de ocuparem a nossa mente com ruminações que nos afastavam de um processamento mais cuidado e detalhado, far-nos-iam comportar de forma mais racional e cuidada. 0 delineamento do estudo partia do paradigma desenvolvido no campo da persuasão, onde se criavam condições experimentais que favorecem a discriminação do modo de processamento subjacente à consideração de mensagens persuasivas. Isto é, perceber se houve um processamento da informação de forma cuidada e sistemática (analisando os 
argumentos apresentados numa mensagem persuasiva) ou um processamento mais superficial que, apenas com base numa "pista persuasiva", permite a geração de uma resposta. Assim, as autoras apresentaram aos participantes argumentos em favor de uma dada posição acompanhados ou não de uma pista persuasiva (credibilidade da fonte), sendo a uns participantes apresentados bons argumentos e a outros maus argumentos (um mau argumento é aqui percebido como um argumento fraco, que induz nos indivíduos pensamentos contrários à posição que pretende sustentar). 0 pressuposto metodológico é de que apenas os participantes que se envolvem num processamento mais profundo da informação recebida irão gerar os tais pensamentos desfavoráveis à posição defendida, não a aceitando. Aqueles que não geram esses pensamentos face a essas mensagens (por estarem a processar de forma superficial a informação recebida) deixar-se-ão influenciar pela mesma num sentido positivo em vez de negativo. Adicionalmente, espera-se que as atitudes destes últimos participantes sejam mais influenciadas pela credibilidade da fonte. Tendo estes dois "barómetros" do modo de processamento em que o participante se envolvia, bastou às autoras dividir os seus participantes em dois grupos: um grupo de participantes que estivesse a sentir-se contente, e outro que estivesse num estado de espírito mais negativo. Tais grupos foram formados a partir da leitura de um de dois textos pré-testados como induzindo o estado de espírito requerido. Os resultados confirmaram a hipótese, demonstrando que apenas os indivíduos num estado de espírito mais negativo resistiram a serem persuadidos com maus argumentos, sendo os mais contentes influenciados pela credibilidade da fonte.

Ao mesmo tempo, na Alemanha, um estudo semelhante era desenvolvido por Norbert Schwarz (Schwarz, 1990) com resultados convergentes. Porém, estes autores apresentavam uma hipótese diferente à de Mackie e Worth (1987). Enquanto estas autoras viam o efeito mediado pelo facto de um estado positivo consumir os recursos cognitivos disponíveis para a tarefa, Schwarz defendia que o efeito era moderado por um aumento da motivação para processar de forma atenta que se verifica nos indivíduos mais tristes (ver também Schwarz \& Bless, 1991). Os que se sentiam contentes utilizavam o seu estado de espírito como informação sobre a posição a ser avaliada.

Desde então, o efeito do estado de espírito no modo de processar tem sido alvo de múltiplas explicações alternativas, que o conceptualizam quer como um efeito indireto (medido por fatores de capacidade e/ou motivação) quer como um efeito direto. 0 efeito tem sido replicado em vários contextos que permitem diferenciar dois modos distintos de processamento (ver Chaiken \& Trope, 1999), tendo-se verificado, por exemplo, que os indivíduos em estados mais positivos utilizam mais heurísticas em processos de tomada de decisão como base para vários tipos de julgamento (Park \& Banaji, 2000; Ruder \& Bless, 2003) e são mais influenciados por estereótipos (Bodenhausen, 1993).

O paradigma que aborda os efeitos do estado de espírito no modo de processar tem, em nossa opinião, as características que definimos como as de um "bom paradigma", como passamos a justificar.

\section{Worth e Mackie (1987) como um "Bom Paradigma Experimental".}

a) O paradigma de Worth e Mackie (1987) desenvolveu-se em contextos adequados de correção de erros. Quase contemporâneos, os estudos de Worth e Mackie e os de Schwarz testam uma hipótese que se confrontava com a ideia de que os estados de humor depressivos deixavam os indivíduos com incapacidade para processar a informação recebida. Para as primeiras autoras, a condição de reversão do efeito é aquela em que fornece mais recursos cognitivos aos participantes contentes; para o segundo, a condição de reversão é aquela em que procura motivá-los para um processamento mais aprofundado. As duas condições demonstram capacidade de anular o efeito do estado de espírito, tendo por isso sido erroneamente assumido pelas duas abordagens que a capacidade/motivação mediariam o efeito. Mantendo o mesmo paradigma, mas associado ao uso de uma estrutura de conhecimento como facilitadora de processamento cognitivo, Bless e colaboradores (Bless et al., 1996) vêm demonstrar que apesar dessas condições moderarem o efeito, o efeito tem também, na verdade, um cariz directo. Tal facto ilustra como a crítica e a correção acompanharam o uso do paradigma para determinar a presença de um efeito e explicar a sua natureza.

b) O paradigma de Worth e Mackie (1987) produz resultados que são apenas fracamente dependentes de uma qualquer teoria. Da apresentação feita do paradigma, fica claro o facto de serem várias as teorias que integram o impacto que o estado de espírito tem no processamento cognitivo como postulado ou suporte ao seu corpo teórico. O efeito inspira o desenvolvimento de teorias explicativas, tendo sido explicado de formas muito diversas. Adicionalmente o efeito impõe restrições ao desenvolvimento de outras teorias, dado que, por exemplo uma teoria dualista (que postule dois modos de processamento) deve incorporar pressupostos que deem conta de porque o estado de espírito é uma variável que modera o processamento. Por ultimo, o efeito não pode ser ignorado por uma teoria que foque a relação que os estados afectivos estabelecem com a cognição.

c) O paradigma de Worth e Mackie (1987) testa hipóteses muito específicas. A hipótese em teste é uma 
e uma única só: quem reporta sentir-se feliz não deve ser sensível ao tipo de argumento persuasivo apresentado (sugerindo um processamento superficial) e quem não o reporta deve ser (sugerindo um processamento mais profundo). Contudo, o paradigma experimental permitia facilmente que os dados fossem os contrários aos por eles postulados. Adicionalmente, deve ter-se em consideração que para além dos artigos acima citados apresentarem estudos que definem o efeito em estudo, eles testam igualmente as condições em que este poderia ser revertido. As operacionalizações destes modos de processar mais superficiais (e.g., uso de estereótipos, uso de heurísticas, uso de scripts) e mais profundos foram muitas e variadas em estudos subsequentes, mas a hipótese seria sempre a mesma. Posteriormente, vários estudos detectam as condições que moderam o efeito (e.g., objectivos de processamento, níveis de motivação, características de personalidade), procurando, com base nelas, delimitá-lo e percebê-lo.

d) O paradigma de Worth e Mackie (1987) representa um teste severo de uma hipótese específica. Apesar de Worth e Mackie (1987) esperarem o efeito oposto, o paradigma experimental permitia facilmente que o padrão de resultados fosse o contrário às suas previsões. Adicionalmente, deve ter-se em consideração que o teste original à hipótese de que o estado de espírito influencia o modo como processamos faz uso do campo onde até à data maior evidência se tinha encontrado a dar suporte à existência de dois modos de processar: o campo da persuasão. Até ao momento, vários estudos tinham referido a influência do estado de espírito no processamento cognitivo (e.g., Isen, 1987). No entanto, nenhum estudo tinha especificado as características de processamento que seriam moderadas pelo estado de espírito. Utilizando dois barómetros independentes, a qualidade dos argumentos e a pista persuasiva (credibilidade da fonte), o estudo exige a identificação dos dois efeitos em simultâneo para ser conclusivo. Mais: o estudo fornece evidência de que os pensamentos dos indivíduos em estado de espírito positivo não medeiam o processo de mudança de atitude, confrontando a hipótese com um teste adicional e com dados convergentes.

Em suma, podemos considerar que o paradigma experimental construído por Worth e Mackie (1986) é um bom paradigma experimental na acepção dada pelo novo experimentalismo. Essa qualidade permitiu que o paradigma ganhasse vida própria e definisse o campo de estudos independentemente da abordagem teórica das autoras. Este paradigma e as suas adaptações posteriores são inevitáveis à teorização sobre os efeitos dos estados afectivos na cognição. Qualquer nova abordagem sobre o tema que queira ser levada a sério tem de ser capaz de oferecer uma explicação para o facto de uma manipulação do estado de espírito induzir os indivíduos a processar diferentemente a informação.

\section{CONCLUSÃO/DISCUSSÃO}

Neste artigo, procurando situar os desenvolvimentos da Cognição Social e o seu contributo para o progresso científico, ilustramos como alguns dos seus paradigmas se definem como "bons paradigmas" na conceção epistemológica que a corrente do Novo Experimentalismo define como essencial ao progresso científico. Os exemplos retirados de diferentes áreas de estudo são representativos de muitos outros paradigmas que se associam à abordagem da cognição social. Parte dos paradigmas desenvolvidos pela Cognição Social são adequações/importações de outras abordagens como as da psicologia Cognitiva (e.g., efeitos de Stroop, primação) e da Psicologia Social (por exemplo, Asch, 1946; Jones \& Harris, 1967) e desenvolvidos com vista a testar hipóteses socio-cognitivas. Outros são desenvolvidos dentro da própria abordagem da Cognição Social (e.g., inferência espontânea de traço; IET, Winter \& Uleman, 1984). A todos se associam efeitos que apesar de poderem necessitar de maior detalhe na sua definição, ou delimitação das variáveis moderadoras, são em si tomados como válidos. Assim sendo, tornam-se incontornáveis em qualquer teoria que se desenvolva sobre o campo. A proliferação destes paradigmas define a Cognição Social como uma abordagem muito frutífera e com um papel que não pode ser ignorado no progresso científico da ciência psicológica.

A abertura desta abordagem a variáveis de natureza neurológica veio criar um novo campo de estudo, e, segundo alguns, definir uma nova disciplina no estudo da psicologia. No desenvolvimento da neuro-psicologia cognitiva parece incontestável o papel da cognição social. Integrando em si o pressuposto de que a compreensão do ser humano passa pela definição dos processos de processamento de informação de estímulos que partilham de forma social o seu significado, a abordagem neuro-sócio-cognitiva permite observar a ativação neuronal que se pressupõe sustentar esses processos. Os seus estudos baseiam-se nos paradigmas desenvolvidos pela abordagem da Cognição Social (e Psicologia Cognitiva) e qualificam os mecanismos neuronais que subjazem aos processos previamente identificados por tais paradigmas. E claro, como acontece com qualquer nova abordagem, assiste-se atualmente ao desenvolvimento de novos e sofisticados paradigmas experimentais e a uma grande expectativa de progresso científico.

Também aqui, o novo experimentalismo oferece critérios para avaliar se os desenvolvimentos obtidos pela abordagem neuro-sócio-cognitiva podem ser considerados verdadeiro progresso científico. Assim, a mera criação de novos paradigmas não pode ser considerada como suficiente para o progresso 
científico. O verdadeiro progresso implica o levar em consideração os "bons experimentos" das abordagens anteriores como restrição à produção teórica. Tal como a Cognição Social incorporou na sua teorização e investigação muitos dos efeitos e paradigmas desenvolvidos na Psicologia Social e na Psicologia Cognitiva, também esta nova abordagem sócio-neuro-cognitiva deve fazer o mesmo, complementando antigas com novas restrições empíricas, ao invés de tentar inventar a pólvora (para um apelo semelhante, ver, por exemplo, Coltheart, 2006). Mas se a abordagem neuro-sócio-cognitiva levar em séria consideração os experimentos com vida própria das outras abordagens, o potencial de criação de restrições teóricas e até de consiliência (quer dizer de convergência entre vários níveis de explicação) é enorme, quer para a nova disciplina quer para as disciplinas que a inspiraram.

\section{REFERÊNCIAS}

Asch, S. E. (1946). Forming impressions of personality. The Journal of Abnormal and Social Psychology, 41(3), 258-290. https://doi.org/10.1037/h0055756

Barsalou, L. (2003). Situated simulation in the human conceptual system. Language and Cognitive Processes, 18(5-6), 513-562. https://doi.org/10.1080/01690960344000026

Bird, A. (2007). What is scientific progress? Noûs, 41(1), 64-89. https://doi.org/10.1111/j.14680068.2007.00638.x

Bless, H., Clore, G. L., Schwarz, N., Golisano, V., Rabe, C., \& Wölk, M. (1996). Mood and the use of scripts: Does a happy mood really lead to mindlessness? Journal of Personality and Social Psychology, 71(4), 665679. https://doi.org/10.1037/0022-3514.71.4.665

Bodenhausen, G. V. (1993). Emotion, arousal and stereotypic judgment: A heuristic model of affect and stereotyping. In D. Mackie \& D. Hamilton (Eds.), Affect, cognition and stereotyping: Interactive processes in intergroup perception (pp. 13-37). Academic Press. https://doi.org/10.1016/b978-008-088579-7.50006-5

Bornstein, R. F. (1989). Exposure and affect: Overview and meta-analysis of research, 1968-1987. Psychological Bulletin, 106(2), 265-289. https://doi.org/10.1037/0033-2909.106.2.265

Bornstein, R. F., \& D'Agostino, P. R. (1992). Stimulus recognition and the mere exposure effect. Journal of Personality and Social Psychology, 63(4), 545-552. https://doi.org/10.1037/0022-3514.63.4.545

Chaiken, S., \& Trope, Y. (Eds.). (1999). Dual-process theories in social psychology. Guilford Press.

Chapman, L. J. (1967). Illusory correlation in observational report. Journal of Verbal Learning and Verbal Behavior, 6(1), 151-155. https://doi.org/10.1016/S0022-5371(67)80066-5

Clark, D. M., Ball, S., \& Pape, D. (1991). An experimental investigation of thought suppression. Behaviour Research and Therapy, 29(3), 253-257. https://doi.org/10.1016/0005-7967(91)90115-j

Coltheart, M. (2006). Perhaps functional neuroimaging has not told us anything about the mind (so far). Cortex, 42(3), 422-427. https://doi.org/10.1016/s0010-9452(08)70374-5

Fiedler, K. (1991). The tricky nature of skewed frequency tables: An information loss account of distinctiveness-based illusory correlations. Journal of Personality and Social Psychology, 60(1), 2436. https://doi.org/10.1037/0022-3514.60.1.24

Forster, J., \& Liberman, N. (2004). A motivational model of post-suppressional rebound. European Review of Social Psychology, 15(1), 1-32. https://doi.org/10.1080/10463280340000081

Freud, S. (1913). The interpretation of dreams. (A. A. Brill, Trans.). The Macmillan Company. (Original work published 1900)

Hamilton, D. L., \& Gifford, R. K. (1976). Illusory correlation in interpersonal perception: A cognitive basis of stereotypic judgments. Journal of Experimental Social Psychology, 12(4), 392-407. https://doi.org/10.1016/S0022-1031(76)80006-6

Hamilton, D. L., Devine, P. G., \& Ostrom, T. M. (1994). Social cognition and classic issues in social psychology. In P. G. Devine, D. L. Hamilton, \& T. M. Ostrom (Eds.), Social cognition: Impact on social psychology (pp. 1-13). Academic Press.

Hamilton, D.L., Dugan, P., \& Trolier, T.K. (1985). The formation of stereotypic beliefs: Further evidence for distinctiveness-based illusory correlations. Journal of Personality and Social Psychology, 48(1), 5-17.

Harmon-Jones, E., \& Allen, J. J. B. (2001). The role of affect in the mere exposure effect: Evidence from psychophysiological and individual differences approaches. Personality and Social Psychology Bulletin, 27(7), 889-898. https://doi.org/10.1177/0146167201277011

Hastie, R., Ostrom, T. M., Ebbensen, E. B., Wyer, R. S., Hamilton, D. L., \& Carlston, D. E. (1980). Person memory. The cognitive basis of social perception. Lawrence Erlbaum Associates.

Isen, A. M. (1987). Positive affect, cognitive processes, and social behavior. Advances in Experimental Social Psychology, 20, 203-253. https://doi.org/10.1016/s0065-2601(08)60415-3 
Jacoby, L. L. (1991). A process dissociation framework: Separating automatic from intentional uses of memory. Journal of Memory and Language, 30(5), 513-541. https://doi.org/10.1016/0749$596 x(91) 90025-f$

Jones, E. E., \& Harris, V. A. (1967). The attribution of attitudes. Journal of Experimental Social Psychology, 3(1), 1-24. https://doi.org/10.1016/0022-1031(67)90034-0

Kuhn, T. S. (1970). The structure of scientific revolutions. University of Chicago Press

Lazarus, R. S. (1984). On the primacy of cognition. American Psychologist, 39(2), 124-129. https://doi.org/10.1037/0003-066X.39.2.124

Macrae, C. N., Bodenhausen, G. V., \& Milne, A. B. (1998). Saying no to unwanted thoughts: Self-focus and the regulation of mental life. Journal of Personality and Social Psychology, 74(3), 578-589. https://doi.org/10.1037/0022-3514.74.3.578

Matlin, M. W. (1971). Response competition, recognition, and affect. Journal of Personality and Social Psychology, 19(3), 295-300. https://doi.org/10.1037/h0031352

Mayo, D. G. (1996). Error and the growth of experimental knowledge. University of Chicago Press

McConnell, A. R., Sherman, S. J., \& Hamilton, D. L. (1994a). Illusory correlation in the perception of groups: An extension of the distinctiveness-based account. Journal of Personality and Social Psychology, 67(3), 414-429 https://doi.org/10.1037//0022-3514.67.3.414

McConnell, A. R., Sherman, S. J., \& Hamilton, D. L. (1994b). On-line and memory-based aspects of individual and group target judgments. Journal of Personality and Social Psychology, 67(2), 173-185. https://doi.org/10.1037/0022-3514.67.2.173

Moreland, R. L., \& Zajonc, R. B. (1977). Is stimulus recognition a necessary condition for the occurrence of exposure effects? Journal of Personality and Social Psychology, 35(4), 191-199. https://doi.org/10.1037/0022-3514.35.4.191

Murphy, S. T., \& Zajonc, R. B. (1993). Affect, cognition, and awareness: Affective priming with optimal and suboptimal stimulus exposures. Journal of Personality and Social Psychology, 64(5), 723-739. https://doi.org/10.1037/0022-3514.64.5.723

Park, J., \& Banaji, M. R. (2000). Mood and heuristics: The influence of happy and sad states on sensitivity and bias in stereotyping. Journal of Personality and Social Psychology, 78(6), 1005-1023. https://doi.org/10.1037/0022-3514.78.6.1005

Popper, K. R. (1963). Conjectures and refutations: The growth of scientific knowledge. Routledge \& Kegan Paul

Putnam, H. (1975). Mind, language, and reality. Cambridge University Press

Richardson-Klavehn, A., \& Bjork, R. A. (1988). Measures of memory. Annual Review of Psychology, 39(1), 475-543. https://doi.org/10.1146/annurev.ps.39.020188.002355

Ruder, M., \& Bless, H. (2003). Mood and the reliance on the ease of retrieval heuristic. Journal of Personality and Social Psychology, 85(1), 20-32. https://doi.org/10.1037/0022-3514.85.1.20

Sanbonmatsu, D. M., Sherman, S. J., \& Hamilton, D. L. (1987). Illusory Correlation in the Perception of Individuals and Groups. Social Cognition, 5(1), 1-25. https://doi.org/10.1521/soco.1987.5.1.1

Sarton, G. (1936). The study of the history of science. Harvard University Press

Schwarz, N. \& Bless, H. (1991) Happy and mindless, but sad and smart? The impact of affective states on analytic reasoning. In J. P. Forgas (Ed.). Emotion and social judgements (pp. 55-70). Pergamon Press.

Schwarz, N. (1990). Feelings as information: Informational and motivational functions of affective states. In E. T. Higgins \& R. Sorrentino (Eds.). Handbook of motivation and cognition: Foundations of social behavior (Vol. 2, pp. 527-561). Guilford.

Sherman, J. W., Kruschke, J. K., Sherman, S. J., Percy, E. J., Petrocelli, J. V., \& Conrey, F. R. (2009). Attentional processes in stereotype formation: A common model for category accentuation and illusory correlation. Journal of Personality and Social Psychology, 96(2), 305-323. https://doi.org/10.1037/a0013778

Stroessner, S. J., \& Plaks, J. E. (2001). Illusory correlation and stereotype formation: Tracing the arc of research over a quarter century. In G. B. Moskowitz (Ed.), Cognitive social psychology: The Princeton symposium on the legacy and future of social cognition (pp. 247-259). Laurence Elrbaum Associates

Wegner, D. M. (1994). Ironic processes of mental control. Psychological Review, 101(1), 34-52. https://doi.org/10.1037/0033-295X.101.1.34

Wegner, D. M., \& Erber, R. (1992). The hyperaccessibility of suppressed thoughts. Journal of Personality and Social Psychology, 63(6), 903-912. https://doi.org/10.1037/0022-3514.63.6.903

Wegner, D. M., \& Gold, D. B. (1995). Fanning old flames: Emotional and cognitive effects of suppressing thoughts of a past relationship. Journal of Personality and Social Psychology, 68(5), 782-792. https://doi.org/10.1037/0022-3514.68.5.782 
Wegner, D. M., Schneider, D. J., Carter, S. R., \& White, T. L. (1987). Paradoxical effects of thought suppression. Journal of Personality and Social Psychology, 53(1), 5. https://doi.org/10.1037//0022-3514.53.1.5

Wenzlaff, R. M., \& Wegner, D. M. (2000). Thought suppression. Annual Review of Winter, L., \& Uleman, J. S. (1984). When are social judgments made? Evidence for the spontaneousness of trait inferences. Journal of Personality and Social Psychology, 47(2), 237-252. https://doi.org/10.1037/00223514.47.2.237

Worth, L. T., \& Mackie, D. M. (1987). Cognitive mediation of positive affect in persuasion. Social Cognition, 5(1), 76-94. https://doi.org/10.1521/soco.1987.5.1.76

Zajonc, R. B. (1968). Attitudinal effects of mere exposure. Journal of Personality and Social Psychology, 9(2, Pt. 2), 1-27. https://doi.org/10.1037/h0025848

Zajonc, R. B. (1980) Feeling and thinking: Preferences need on inferences. American Psychologist, 35(2), 151-175. https://doi.org/10.1037/0003-066x.35.2.151

$\begin{array}{ll}\text { Historial do artigo } & \\ \text { Recebido } & 12 / 2019 \\ \text { Aceite } & 11 / 2020 \\ \text { Publicado online } & 03 / 2021 \\ \text { Publicado } & 06 / 2021\end{array}$

